\title{
A characterization of strict concordance relations*
}

\author{
D. Bouyssou ${ }^{\dagger}$ \\ M. Pirlot $\ddagger$ \\ CNRS - LAMSADE Faculté Polytechnique de Mons \\ 29 March 2001 - Revised version
}

\begin{abstract}
Based on a general framework for conjoint measurement that allows for intransitive preferences, this paper proposes a characterization of "strict concordance relations". This characterization shows that the originality of such relations lies in their very crude way to distinguish various levels of "preference differences" on each attribute.
\end{abstract}

Keywords: MCDM, Conjoint Measurement, Nontransitive Preferences, Outranking Methods, Concordance Relations.

\section{Introduction}

A basic problem in the field of Multiple Criteria Decision Making (MCDM) is to build a preference relation on a set of alternatives evaluated on several attributes on the basis of preferences expressed on each attribute and interattribute information such as weights or trade-offs.

*We wish to thank Patrice Perny for his helpful comments on an earlier draft of this text. The usual caveat applies.

†LAMSADE, Université Paris Dauphine, Place du Maréchal de Lattre de Tassigny, F-75775 Paris Cedex 16, France, tel: +33 1440548 98, fax: +33 1440540 91, e-mail: bouyssou@lamsade.dauphine.fr.

${ }^{\ddagger}$ Faculté Polytechnique de Mons, 9, rue de Houdain, B-7000 Mons, Belgium, e-mail: pirlot@mathro.fpms.ac.be. 
B. Roy proposed several outranking methods [Roy68, Roy96b, RB93, Vin92, Vin99, Bou01] as alternatives to the dominant value function approach [Fis70, KR76, Wak89]. In outranking methods, the construction of a preference relation is based on pairwise comparisons of the alternatives. This preference relation may either be reflexive as in the ELECTRE methods [Roy91] (it is then interpreted as an "at least as good" relation) or asymmetric as in TACTIC [Van86] (it is then interpreted as a "strict preference" relation). Most outranking methods, including ELECTRE and TACTIC, make use of the so-called concordance-discordance principle which consists in accepting a preferential assertion linking an alternative $a$ to an alternative $b$ if:

- Concordance Condition: a majority of the attributes supports this assertion and if,

- Non-Discordance Condition: the opposition of the other attributes is not "too strong".

In this paper we restrict our attention to outranking methods such as TACTIC aiming at building a crisp (i.e. nonfuzzy) asymmetric preference relation. Based on a general framework for conjoint measurement that allows for intransitive preferences [BP00], we propose a characterization of "strict concordance relations", i.e. asymmetric binary relations resulting from the application of the concordance condition in such methods. This characterization shows that the essential distinctive feature of these relations lies in their very crude way to distinguish various levels of "preference differences" on each attribute.

This paper is organized as follows. In section 2, we briefly recall some notions on outranking relations and define "strict concordance relations". Section 3 presents our general framework for conjoint measurement that allows for intransitive preferences. This framework is used in section 4 to characterize strict concordance relations. A final section discusses our findings and indicates directions for future research. Throughout the paper, unless otherwise mentioned, we follow the terminology of [Bou96] concerning binary relations. 


\section{Outranking methods leading to an asym- metric relation}

\subsection{TACTIC [Van86]}

Consider two alternatives $x$ and $y$ evaluated on a family $N=\{1,2, \ldots, n\}$ of attributes. A first step in the comparison of $x=\left(x_{1}, x_{2}, \ldots, x_{n}\right)$ and $y=\left(y_{1}, y_{2}, \ldots, y_{n}\right)$ is to know how they compare on each attribute. In TACTIC, it is supposed that evaluations on an attribute can be compared using an asymmetric binary relation $P_{i}$ that is a strict semiorder (i.e. an irreflexive, Ferrers and semi-transitive relation). The asymmetry of $P_{i}$ implies that one and only one of the following propositions is true: $x_{i} P_{i} y_{i}$ or $y_{i} P_{i} x_{i}$ or $x_{i} I_{i} y_{i}$ (i.e. $\operatorname{Not}\left[x_{i} P_{i} y_{i}\right]$ and $\operatorname{Not}\left[y_{i} P_{i} x_{i}\right]$ )

When comparing $x$ to $y$, the following subsets of attributes play a vital part in TACTIC:

$$
\begin{gathered}
P(x, y)=\left\{i \in N: x_{i} P_{i} y_{i}\right\} \\
I(x, y)=I(y, x)=\left\{i \in N: x_{i} I_{i} y_{i}\right\} \text { and } \\
P(y, x)=\left\{i \in N: y_{i} P_{i} x_{i}\right\} .
\end{gathered}
$$

Since $P_{i}$ is asymmetric, we have $P(x, y) \cap P(y, x)=\emptyset$. Note that, by construction, $I(x, y)=I(y, x), P(x, y) \cap I(x, y)=\emptyset$ and $P(x, y) \cup I(x, y) \cup P(y, x)=$ $N$.

In its concordance part, TACTIC declares that $x$ is preferred to $y(x \mathcal{P} y)$ if the attributes in $P(x, y)$ are "strictly more important" than the attributes in $P(y, x)$. Since it appears impractical to completely assess an importance relation between all disjoint subsets of attributes, TACTIC assigns a weight to each attribute and supposes that the importance of a subset of attributes is derived additively. More precisely, if $w_{i}>0$ is the weight assigned to attribute $i \in N$, we have in the concordance part of TACTIC:

$$
x \mathcal{P} y \Leftrightarrow \sum_{i \in P(x, y)} w_{i}>\rho \sum_{j \in P(y, x)} w_{j}
$$

where $\rho \geq 1$ is a concordance threshold.

The preceding analysis based on concordance does not take into account the magnitude of the preference differences between the evaluations of $x$ and $y$ on each attribute besides the distinction between "positive", "negative" and "neutral" differences. This may be criticized since, if on some $j \in P(y, x)$ 
the difference of preference in favor of $y$ is "very large", it may be risky to conclude that $x \mathcal{P} y$ even if the attributes in $P(x, y)$ are strictly more important than the attributes in $P(y, x)$. This leads to the discordance part of the method. The idea of very large preference differences is captured through a strict semiorder $V_{i} \subseteq P_{i}$ on each attribute $i \in N$ and the discordance part of the method forbids to have $x \mathcal{P} y$ whenever $y_{j} V_{j} x_{j}$, for some $j \in P(y, x)$. In summary, we have in TACTIC:

$$
\begin{gathered}
x \mathcal{P} y \Leftrightarrow \\
\sum_{i \in P(x, y)} w_{i}>\rho \sum_{j \in P(y, x)} w_{j} \\
\text { and } \\
N o t\left[y_{j} V_{j} x_{j}\right] \text { for all } j \in P(y, x)
\end{gathered}
$$

where $P_{i}$ and $V_{i}$ are strict semiorders such that $V_{i} \subseteq P_{i}, w_{i}>0$ and $\rho \geq 1$. We refer to [Van86] for a thorough analysis of this method including possible assessment techniques for $P_{i}, V_{i}, w_{i}$ and $\rho$.

Simple examples show that, in general, a relation $\mathcal{P}$ built using (1) or (2) may not be transitive and may even contain circuits. The use of such a relation $\mathcal{P}$ for decision-aid purposes therefore calls for the application of specific techniques, see [Roy91, RB93, Van90].

\subsection{Strict concordance relations}

Relation (1) is only one among the many possible ways to implement the concordance principle in order to build an asymmetric relation. The following elements appear central in the analysis:

- an asymmetric relation $P_{i}$ on each $X_{i}$ allowing to partition $N$ into $P(x, y), P(y, x)$ and $I(x, y)$,

- an asymmetric importance relation $\triangleright$ between disjoint subsets of attributes, allowing to compare $P(x, y)$ and $P(y, x)$, which is monotonic (with respect to inclusion), i.e. such that:

$$
[A \triangleright B, C \supseteq A, B \supseteq D, C \cap D=\emptyset] \Rightarrow[C \triangleright D] .
$$

This motivates the following, inspired by [FPng]: 
Definition 1 (Strict concordance relations)

Consider a set $Y \subseteq X_{1} \times X_{2} \times \cdots \times X_{n}$ of alternatives evaluated on a set $N=\{1,2, \ldots, n\}$ of attributes. A binary relation $\mathcal{P}$ on $Y$ is said to be a strict concordance relation if there are:

- an asymmetric binary relation $\triangleright$ between disjoint subsets of $N$ that is monotonic and,

- an asymmetric binary relation $P_{i}$ on each $X_{i}(i=1,2, \ldots, n)$,

such that, for all $x, y \in Y$ :

$$
x \mathcal{P} y \Leftrightarrow P(x, y) \triangleright P(y, x),
$$

where $P(x, y)=\left\{i \in N: x_{i} P_{i} y_{i}\right\}$.

It should be clear that any binary relation built using (1) is a strict concordance relation.

The above definition does only require the asymmetry of the relations $P_{i}$. Although this it is at variance with what is done in most outranking methods ( $P_{i}$ generally being strict semiorders), this additional generality will prove to have little impact in what follows. We defer to section 5 the discussion of a possible introduction of discordance in our analysis.

We already noticed with TACTIC that $\mathcal{P}$ may be a strict concordance relation without being transitive or without circuit. This does not imply that, for a given number of attributes and a given set of alternatives, any asymmetric relation is a strict concordance relation. The purpose of this paper is to provide a characterization of such relations when the set of alternatives is rich, i.e. when $Y=X=X_{1} \times X_{2} \times \cdots \times X_{n}$ ([Bou96] studies the, simpler, case in which the number of attributes is not fixed).

\section{A general framework for nontransitive conjoint measurement}

In the rest of this paper, we always consider a set $X=\prod_{i=1}^{n} X_{i}$ with $n \geq 2$; elements of $X$ will be interpreted as alternatives evaluated on a set $N=\{1,2, \ldots, n\}$ of attributes. Unless otherwise stated, in order to avoid unnecessary complications, we suppose throughout that $X$ is finite. When $J \subseteq N$, we denote by $X_{J}$ (resp. $\left.X_{-J}\right)$ the set $\prod_{i \in J} X_{i}\left(\operatorname{resp} . \prod_{i \notin J} X_{i}\right)$. With 
customary abuse of notation, $\left(x_{J}, y_{-J}\right)$ will denote the element $w \in X$ such that $w_{i}=x_{i}$ if $i \in J$ and $w_{i}=y_{i}$ otherwise (when $J=\{i\}$ we simply write $X_{-i}$ and $\left.\left(x_{i}, y_{-i}\right)\right)$.

Let $\succ$ be a binary relation on $X$ interpreted as "strict preference". The absence of strict preference is denoted by $\sim$ (i.e. $x \sim y \Leftrightarrow \operatorname{Not}[x \succ y]$ and $N o t[y \succ x])$ and we define $\succsim$ on $X$ letting $x \succsim y \Leftrightarrow[x \succ y$ or $x \sim y]$. We define the following binary relations on $X_{J}$ with $J \subseteq N$ :

$$
\begin{gathered}
x_{J} \succ_{J} y_{J} \text { iff }\left(x_{J}, z_{-J}\right) \succ\left(y_{J}, z_{-J}\right), \text { for all } z_{-J} \in X_{-J}, \\
x_{J} \succ_{J}^{\circ} y_{J} \text { iff }\left(x_{J}, z_{-J}\right) \succ\left(y_{J}, z_{-J}\right), \text { for some } z_{-J} \in X_{-J},
\end{gathered}
$$

where $x_{J}, y_{J} \in X_{J}$ (when $J=\{i\}$ we write $\succ_{i}$ instead of $\succ_{\{i\}}$ ).

If, for all $x_{J}, y_{J} \in X_{J}, x_{J} \succ_{J}^{\circ} y_{J}$ implies $x_{J} \succ_{J} y_{J}$, we say that $\succ$ is independent for $J$. If $\succ$ is independent for all nonempty subsets of attributes we say that $\succ$ is independent. It is not difficult to see that a binary relation is independent if and only if it is independent for $N \backslash\{i\}$, for all $i \in N$, see e.g. [Wak89].

We say that attribute $i \in N$ is influent (for $\succ$ ) if there are $x_{i}, y_{i}, z_{i}, w_{i} \in$ $X_{i}$ and $x_{-i}, y_{-i} \in X_{-i}$ such that $\left(x_{i}, x_{-i}\right) \succ\left(y_{i}, y_{-i}\right)$ and $\operatorname{Not}\left[\left(z_{i}, x_{-i}\right) \succ\right.$ $\left.\left(w_{i}, y_{-i}\right)\right]$ and degenerate otherwise. It is clear that a degenerate attribute has no influence whatsoever on the comparison of the elements of $X$ and may be suppressed from $N$.

We say that attribute $i \in N$ is essential (for $\succ$ ) if $\succ_{i}$ is not empty. It should be clear that any essential attribute is influent. The converse does not hold however. It will not be supposed here that all attributes are essential.

We envisage in this section relations $\succ$ that can be represented as:

$$
x \succ y \Leftrightarrow F\left(p_{1}\left(x_{1}, y_{1}\right), p_{2}\left(x_{2}, y_{2}\right), \ldots, p_{n}\left(x_{n}, y_{n}\right)\right)>0
$$

where $p_{i}$ are real-valued functions on $X_{i}^{2}$ that are skew symmetric (i.e. such that $p_{i}\left(x_{i}, y_{i}\right)=-p_{i}\left(y_{i}, x_{i}\right)$, for all $\left.x_{i}, y_{i} \in X_{i}\right)$ and $F$ is a real-valued function on $\prod_{i=1}^{n} p_{i}\left(X_{i}^{2}\right)$ being nondecreasing in all its arguments and odd (i.e. such that $F(\mathbf{x})=-F(-\mathbf{x})$, abusing notations in an obvious way). We summarize some useful properties of model $(\mathrm{M})$ in the following:

Proposition 1 If $\succ$ satisfies model $(M)$ then:

$i$. $\succ$ is asymmetric and independent, 
ii. $\left[x_{i} \succ_{i} y_{i}\right.$ for all $\left.i \in J \subseteq N\right] \Rightarrow\left[x_{J} \succ_{J} y_{J}\right]$.

\section{Proof of proposition 1}

$i$. The asymmetry of $\succ$ follows from the skew symmetry of all $p_{i}$ and the oddness of $F$. Since $p_{i}\left(x_{i}, x_{i}\right)=0$, the independence of $\succ$ follows.

ii. Observe that $x_{i} \succ_{i} y_{i}$ is equivalent to $F\left(p_{i}\left(x_{i}, y_{i}\right), \mathbf{0}\right)>0$ (using obvious notations). Since $F(\mathbf{0})=0$, the nondecreasingness of $F$ leads to $p_{i}\left(x_{i}, y_{i}\right)>0$. The desired property easily follows using the nondecreasingness of $F$.

Two conditions, inspired by [BP00], will prove useful for the analysis of model (M). Let $\succ$ be a binary relation on a set $X=\prod_{i=1}^{n} X_{i}$. This relation is said to satisfy:

$A R C 1_{i}$ if

$$
\left.\begin{array}{c}
\left(x_{i}, a_{-i}\right) \succ\left(y_{i}, b_{-i}\right) \\
\text { and } \\
\left(z_{i}, c_{-i}\right) \succ\left(w_{i}, d_{-i}\right)
\end{array}\right\} \Rightarrow\left\{\begin{array}{l}
\left(x_{i}, c_{-i}\right) \succ\left(y_{i}, d_{-i}\right) \\
\text { or } \\
\left(z_{i}, a_{-i}\right) \succ\left(w_{i}, b_{-i}\right),
\end{array}\right.
$$

$A R C 2_{i}$ if

$$
\left.\begin{array}{l}
\left(x_{i}, a_{-i}\right) \succ\left(y_{i}, b_{-i}\right) \\
\text { and } \\
\left(y_{i}, c_{-i}\right) \succ\left(x_{i}, d_{-i}\right)
\end{array}\right\} \Rightarrow\left\{\begin{array}{l}
\left(z_{i}, a_{-i}\right) \succ\left(w_{i}, b_{-i}\right) \\
\text { or } \\
\left(w_{i}, c_{-i}\right) \succ\left(z_{i}, d_{-i}\right),
\end{array}\right.
$$

for all $x_{i}, y_{i}, z_{i}, w_{i} \in X_{i}$ and all $a_{-i}, b_{-i}, c_{-i}, d_{-i} \in X_{-i}$. We say that $\succ$ satisfies $A R C 1$ (resp. $A R C 2$ ) if it satisfies $A R C 1_{i}$ (resp. $A R C 2_{i}$ ) for all $i \in N$.

Condition $A R C 1_{i}$ (Asymmetric inteR-attribute Cancellation) suggests that $\succ$ induces on $X_{i}^{2}$ a relation that compares "preference differences" in a well-behaved way: if $\left(x_{i}, y_{i}\right)$ is a larger preference difference than $\left(z_{i}, w_{i}\right)$ and $\left(z_{i}, c_{-i}\right) \succ\left(w_{i}, d_{-i}\right)$ then we should have $\left(x_{i}, c_{-i}\right) \succ\left(y_{i}, d_{-i}\right)$ and vice versa. The idea that the comparison of preference differences is central to the analysis of conjoint measurement models was powerfully stressed by [Wak88, Wak89].

Condition $A R C 2_{i}$ suggests that the preference difference $\left(x_{i}, y_{i}\right)$ is linked to the "opposite" preference difference $\left(y_{i}, x_{i}\right)$. It says that if the preference difference between $z_{i}$ and $w_{i}$ is not larger than the preference difference between $x_{i}$ and $y_{i}$ then the preference difference between $w_{i}$ and $z_{i}$ should 
be larger than the preference difference between $y_{i}$ and $x_{i}$. Taking $x_{i}=y_{i}$, $z_{i}=w_{i}, a_{-i}=c_{-i}$ and $b_{-i}=d_{-i}$ shows that $A R C 2_{i}$ implies that $\succ$ is independent for $N \backslash\{i\}$ and, hence, independent.

The following lemma shows that these two conditions are independent and necessary for model (M).

\section{Lemma 1}

i. Model (M) implies ARC1 and ARC2,

ii. In the class of asymmetric relations, $A R C 1$ and $A R C 2$ are independent conditions.

\section{Proof of lemma 1}

$i$. Suppose that $\left(x_{i}, a_{-i}\right) \succ\left(y_{i}, b_{-i}\right)$ and $\left(z_{i}, c_{-i}\right) \succ\left(w_{i}, d_{-i}\right)$. Using model (M) we have:

$$
F\left(p_{i}\left(x_{i}, y_{i}\right),\left(p_{j}\left(a_{j}, b_{j}\right)\right)_{j \neq i}\right)>0
$$

and

$$
F\left(p_{i}\left(z_{i}, w_{i}\right),\left(p_{j}\left(c_{j}, d_{j}\right)\right)_{j \neq i}\right)>0
$$

abusing notations in an obvious way.

If $p_{i}\left(x_{i}, y_{i}\right) \geq p_{i}\left(z_{i}, w_{i}\right)$ then using the nondecreasingness of $F$, we have $F\left(p_{i}\left(x_{i}, y_{i}\right),\left(p_{j}\left(c_{j}, d_{j}\right)\right)_{j \neq i}\right)>0$ so that $\left(x_{i}, c_{-i}\right) \succ\left(y_{i}, d_{-i}\right)$. If $p_{i}\left(z_{i}, w_{i}\right)>$ $p_{i}\left(x_{i}, y_{i}\right)$ we have $F\left(p_{i}\left(z_{i}, w_{i}\right),\left(p_{j}\left(a_{j}, b_{j}\right)\right)_{j \neq i}\right)>0$ so that $\left(z_{i}, a_{-i}\right) \succ\left(w_{i}, b_{-i}\right)$. Hence $A R C 1$ holds.

Similarly, suppose that $\left(x_{i}, a_{-i}\right) \succ\left(y_{i}, b_{-i}\right)$ and $\left(y_{i}, c_{-i}\right) \succ\left(x_{i}, d_{-i}\right)$. We thus have:

$$
F\left(p_{i}\left(x_{i}, y_{i}\right),\left(p_{j}\left(a_{j}, b_{j}\right)\right)_{j \neq i}\right)>0
$$

and

$$
F\left(p_{i}\left(y_{i}, x_{i}\right),\left(p_{j}\left(c_{j}, d_{j}\right)\right)_{j \neq i}\right)>0 .
$$

If $p_{i}\left(x_{i}, y_{i}\right) \geq p_{i}\left(z_{i}, w_{i}\right)$, the skew symmetry of $p_{i}$ implies $p_{i}\left(w_{i}, z_{i}\right) \geq p_{i}\left(y_{i}, x_{i}\right)$. Using the nondecreasingness of $F$ we have $F\left(p_{i}\left(w_{i}, z_{i}\right),\left(p_{j}\left(c_{j}, d_{j}\right)\right)_{j \neq i}\right)>0$ so that $\left(w_{i}, c_{-i}\right) \succ\left(z_{i}, d_{-i}\right)$. Similarly, if $p_{i}\left(z_{i}, w_{i}\right)>p_{i}\left(x_{i}, y_{i}\right)$ we have, using the nondecreasingness of $F, F\left(p_{i}\left(z_{i}, w_{i}\right),\left(p_{j}\left(a_{j}, b_{j}\right)\right)_{j \neq i}\right)>0$ so that $\left(z_{i}, a_{-i}\right) \succ$ $\left(w_{i}, b_{-i}\right)$. Hence $A R C 2$ holds.

$i i$. It is easy to build asymmetric relations violating $A R C 1$ and $A R C 2$. Using theorem 1 below, it is clear that there are asymmetric relations satisfying both $A R C 1$ and $A R C 2$. We provide here the remaining two examples. 
1. Let $X=\{a, b, c\} \times\{x, y, z\}$ and let $\succ$ on $X$ be empty except that $(a, x) \succ(b, y)$ and $(a, x) \succ(c, z)$. Relation $\succ$ is asymmetric. Since $\operatorname{Not}[(a, x) \succ(b, z)]$ and $\operatorname{Not}[(a, x) \succ(c, y)], \succ$ violates $A R C 1$. Condition $A R C 2$ is trivially satisfied.

2. Let $X=\{a, b\} \times\{x, y\}$ and $\succ$ on $X$ be empty except that $(a, x) \succ$ $(a, y)$. It is clear that $\succ$ is asymmetric but not independent, so that $A R C 2$ is violated. Condition $A R C 1$ is trivially satisfied.

In order to interpret conditions $A R C 1$ and $A R C 2$ in terms of preference differences, we define the binary relations $\succsim_{i}^{*}$ and $\succsim_{i}^{* *}$ on $X_{i}^{2}$ letting, for all $x_{i}, y_{i}, z_{i}, w_{i} \in X_{i}$,

$$
\begin{aligned}
& \left(x_{i}, y_{i}\right) \succsim_{i}^{*}\left(z_{i}, w_{i}\right) \Leftrightarrow \\
& \quad\left[\text { for all } a_{-i}, b_{-i} \in X_{-i},\left(z_{i}, a_{-i}\right) \succ\left(w_{i}, b_{-i}\right) \Rightarrow\left(x_{i}, a_{-i}\right) \succ\left(y_{i}, b_{-i}\right)\right]
\end{aligned}
$$

and

$$
\left(x_{i}, y_{i}\right) \succsim_{i}^{* *}\left(z_{i}, w_{i}\right) \Leftrightarrow\left[\left(x_{i}, y_{i}\right) \succsim_{i}^{*}\left(z_{i}, w_{i}\right) \text { and }\left(w_{i}, z_{i}\right) \succsim_{i}^{*}\left(y_{i}, x_{i}\right)\right] .
$$

It is easy to see that $\succsim_{i}^{*}$ (and, hence, $\succsim_{i}^{* *}$ ) is transitive by construction and that the symmetric parts of these relations $\left(\sim_{i}^{*}\right.$ and $\left.\sim_{i}^{* *}\right)$ are equivalence relations (the hypothesis that attribute $i \in N$ is influent meaning that $\sim_{i}^{*}$ has at least two distinct equivalence classes). Observe that, by construction, $\succsim_{i}^{* *}$ is reversible, i.e. $\left(x_{i}, y_{i}\right) \succsim_{i}^{* *}\left(z_{i}, w_{i}\right) \Leftrightarrow\left(w_{i}, z_{i}\right) \succsim_{i}^{* *}\left(y_{i}, x_{i}\right)$.

The consequences of $A R C 1_{i}$ and $A R C 2_{i}$ on relations $\succsim_{i}^{*}$ and $\succsim_{i}^{* *}$ are noted in the following lemma; we omit its straightforward proof.

\section{Lemma 2}

$$
\begin{aligned}
& \text { i. } A R C 1_{i} \Leftrightarrow\left[\succsim_{i}^{*} \text { is complete }\right], \\
& \text { ii. } A R C 2_{i} \Leftrightarrow \\
& \quad\left[\text { for all } x_{i}, y_{i}, z_{i}, w_{i} \in X_{i}, N o t\left[\left(x_{i}, y_{i}\right) \succsim_{i}^{*}\left(z_{i}, w_{i}\right)\right] \Rightarrow\left(y_{i}, x_{i}\right) \succsim_{i}^{*}\left(w_{i}, z_{i}\right)\right], \\
& \text { iii. }\left[A R C 1_{i} \text { and } A R C 2_{i}\right] \Leftrightarrow\left[\succsim_{i}^{* *} \text { is complete }\right] .
\end{aligned}
$$

For the sake of easy reference, we note a few useful connections between $\succsim_{i}^{*}, \succsim_{i}^{* *}$ and $\succ$ in the following lemma. 
Lemma 3 For all $x, y \in X$ and all $z_{i}, w_{i} \in X_{i}$,

$$
\begin{aligned}
\text { i. } & {\left[x \succ y \text { and }\left(z_{i}, w_{i}\right) \succsim_{i}^{*}\left(x_{i}, y_{i}\right)\right] \Rightarrow\left(z_{i}, x_{-i}\right) \succ\left(w_{i}, y_{-i}\right), } \\
\text { ii. } & {\left[\left(z_{i}, w_{i}\right) \sim_{i}^{*}\left(x_{i}, y_{i}\right) \text { for all } i \in N\right] \Rightarrow[x \succ y \Leftrightarrow z \succ w] } \\
\text { iii. } & {\left[x \succsim y \text { and }\left(z_{i}, w_{i}\right) \succsim_{i}^{* *}\left(x_{i}, y_{i}\right) \Rightarrow\left(z_{i}, x_{-i}\right) \succsim\left(w_{i}, y_{-i}\right)\right] } \\
\text { iv. } & {\left[\left(z_{i}, w_{i}\right) \sim_{i}^{* *}\left(x_{i}, y_{i}\right) \text { for all } i \in N\right] \Rightarrow[x \succ y \Leftrightarrow z \succ w] \text { and }[y \succ x \Leftrightarrow} \\
& w \succ z] .
\end{aligned}
$$

\section{Proof of lemma 3}

$i$. is obvious from the definition of $\succsim_{i}^{*}$ and $i i$. is immediate from $i$.

iii. Suppose that $x \sim y,\left(z_{i}, w_{i}\right) \succsim_{i}^{* *}\left(x_{i}, y_{i}\right)$ and $\left(w_{i}, y_{-i}\right) \succ\left(z_{i}, x_{-i}\right)$. By hypothesis, we have $\operatorname{Not}\left[\left(y_{i}, y_{-i}\right) \succ\left(x_{i}, x_{-i}\right)\right]$. Since $\left(w_{i}, y_{-i}\right) \succ\left(z_{i}, x_{-i}\right)$, this implies $\operatorname{Not}\left[\left(y_{i}, x_{i}\right) \succsim_{i}^{*}\left(w_{i}, z_{i}\right)\right]$. Since $A R C 1$ and $A R C 2$ hold, we know that $\succsim_{i}^{* *}$ is complete so that $\left(w_{i}, z_{i}\right) \succ_{i}^{* *}\left(y_{i}, x_{i}\right)$, a contradiction. Part $i v$. is immediate from ii. and iii.

For finite or countably infinite sets $X$ conditions $A R C 1, A R C 2$ combined with asymmetry allow to characterize model (M). We have:

Theorem 1 Let $\succ$ be a binary relation on a finite or countably infinite set $X=\prod_{i=1}^{n} X_{i}$. Then $\succ$ satisfies model $(M)$ iff it is asymmetric and satisfies $A R C 1$ and $A R C 2$.

\section{Proof of theorem 1}

Necessity results from lemma 1 and proposition 1 . We establish sufficiency below.

Since $A R C 1_{i}$ and $A R C 2_{i}$ hold, we know from lemma 2 that $\succsim_{i}^{* *}$ is complete so that it is a weak order. This implies that $\succsim_{i}^{*}$ is a weak order and, since $X$ is finite or countably infinite, there is a real-valued function $q_{i}$ on $X_{i}^{2}$ such that, for all $x_{i}, y_{i}, z_{i}, w_{i} \in X_{i},\left(x_{i}, y_{i}\right) \succsim_{i}^{*}\left(z_{i}, w_{i}\right) \Leftrightarrow$ $q_{i}\left(x_{i}, y_{i}\right) \geq q_{i}\left(z_{i}, w_{i}\right)$. Given a particular numerical representation $q_{i}$ of $\succsim_{i}^{*}$, let $p_{i}\left(x_{i}, y_{i}\right)=q_{i}\left(x_{i}, y_{i}\right)-q_{i}\left(y_{i}, x_{i}\right)$. It is obvious that $p_{i}$ is skew symmetric and represents $\succsim_{i}^{* *}$.

Define $F$ as follows:

$$
\begin{aligned}
& F\left(p_{1}\left(x_{1}, y_{1}\right), p_{2}\left(x_{2}, y_{2}\right), \ldots, p_{n}\left(x_{n}, y_{n}\right)\right)= \\
& \left\{\begin{array}{l}
f\left(g\left(p_{1}\left(x_{1}, y_{1}\right), p_{2}\left(x_{2}, y_{2}\right), \ldots, p_{n}\left(x_{n}, y_{n}\right)\right)\right) \text { if } x \succ y, \\
0 \text { if } x \sim y, \\
-f\left(-g\left(p_{1}\left(x_{1}, y_{1}\right), p_{2}\left(x_{2}, y_{2}\right), \ldots, p_{n}\left(x_{n}, y_{n}\right)\right)\right) \text { otherwise },
\end{array}\right.
\end{aligned}
$$


where $g$ is any function from $\mathbb{R}^{n}$ to $\mathbb{R}$ increasing in all its arguments and odd (e.g. $\Sigma$ ) and $f$ is any increasing function from $\mathbb{R}$ into $(0,+\infty)$ (e.g. $\exp (\cdot)$ or $\left.\arctan (\cdot)+\frac{\pi}{2}\right)$.

The well-definedness of $F$ follows from part $i v$. of lemma 3 and the definition of the $p_{i}$ 's. It is odd by construction.

To show that $F$ is nondecreasing, suppose that $p_{i}\left(z_{i}, w_{i}\right)>p_{i}\left(x_{i}, y_{i}\right)$, i.e. that $\left(z_{i}, w_{i}\right) \succ_{i}^{* *}\left(x_{i}, y_{i}\right)$. If $x \succ y$, we know from part $i$. of lemma 3 that $\left(z_{i}, x_{-i}\right) \succ\left(w_{i}, y_{-i}\right)$ and the conclusion follows from the definition of $F$. If $x \sim y$, we know from part iii. of lemma 3 that $\operatorname{Not}\left[\left(w_{i}, y_{-i}\right) \succ\left(z_{i}, x_{-i}\right)\right]$ and the conclusion follows from the definition of $F$. If $y \succ x$ we have either $\left(w_{i}, y_{-i}\right) \succ\left(z_{i}, x_{-i}\right)$ or $\left(z_{i}, x_{-i}\right) \succsim\left(w_{i}, y_{-i}\right)$. In either case, the conclusion follows from the definition of $F$.

Following [BP00], it is not difficult to extend this result to sets of arbitrary cardinality adding a, necessary, condition implying that the weak orders $\succsim_{i}^{* *}$ have a numerical representation. It should be observed that model (M) seems sufficiently general to contain as particular cases most conjoint measurement models including: additive utilities [KLST71, Wak89], additive differences [Tve69, Fis92] and additive nontransitive models [Bou86, Fis90b, Fis90a, Fis91, Vin91]. We show in the next section that it also contains strict concordance relations.

It should be observed that in model $(\mathrm{M})$, the function $p_{i}$ does not necessarily represent $\succsim_{i}^{* *}$. It is however easy to see that we always have:

$$
\left(x_{i}, y_{i}\right) \succ_{i}^{* *}\left(z_{i}, w_{i}\right) \Rightarrow p_{i}\left(x_{i}, y_{i}\right)>p_{i}\left(z_{i}, w_{i}\right) .
$$

Hence $\left|p_{i}\left(X_{i}^{2}\right)\right|$ is an upper bound for the number of equivalence classes of $\succsim_{i}^{* *}$

\section{A characterization of strict concordance relations}

Our main result in this section says that all strict concordance relations (definition 1 ) can be represented in model $(\mathrm{M})$ with relations $\succsim_{i}^{* *}$ having at most three equivalence classes and vice versa.

Theorem 2 The following are equivalent: 
i. $\succ$ has a representation in model $(M)$ with all relations $\succsim_{i}^{* *}$ having at most three distinct equivalence classes,

ii. $\succ$ is a strict concordance relation.

\section{Proof of theorem 2}

$i i \Rightarrow i$. Given equation (4), the claim will be proven if we build a representation of $\succ$ in model $(\mathrm{M})$ with functions $p_{i}$ taking only three distinct values. Define $p_{i}$ as:

$$
p_{i}\left(x_{i}, y_{i}\right)=\left\{\begin{array}{l}
1 \text { if } x_{i} P_{i} y_{i} \\
0 \text { if } x_{i} I_{i} y_{i} \\
-1 \text { if } y_{i} P_{i} x_{i}
\end{array}\right.
$$

Since $P_{i}$ is asymmetric, the function $p_{i}$ is well-defined and skew-symmetric.

Define $F$ letting:

$$
F\left(p_{1}\left(x_{1}, y_{1}\right), p_{2}\left(x_{2}, y_{2}\right), \ldots, p_{n}\left(x_{n}, y_{n}\right)\right)=\left\{\begin{array}{l}
1 \text { if } x \succ y, \\
-1 \text { if } y \succ x \\
0 \text { otherwise. }
\end{array}\right.
$$

Since, by hypothesis, $[P(x, y)=P(z, w)$ and $P(y, x)=P(w, z)] \Rightarrow[x \succ$ $y \Leftrightarrow z \succ w]$, it is easy to see that $F$ is well-defined. It is clearly odd. The monotonicity of $\triangleright$ implies that $F$ is nondecreasing in all its arguments.

$i \Rightarrow i$. Define $P_{i}$ letting, for all $x_{i}, y_{i} \in X_{i}, x_{i} P_{i} y_{i} \Leftrightarrow\left(x_{i}, y_{i}\right) \succ_{i}^{* *}\left(y_{i}, y_{i}\right)$.

Suppose that $x_{i} P_{i} y_{i}$ and $y_{i} P_{i} x_{i}$ so that $\left(x_{i}, y_{i}\right) \succ_{i}^{* *}\left(y_{i}, y_{i}\right)$ and $\left(y_{i}, x_{i}\right) \succ_{i}^{* *}$ $\left(x_{i}, x_{i}\right)$. Since $\succ$ is independent, we have $\left(y_{i}, y_{i}\right) \sim_{i}^{* *}\left(x_{i}, x_{i}\right)$ so that $\left(y_{i}, x_{i}\right) \succ_{i}^{* *}$ $\left(y_{i}, y_{i}\right)$. The reversibility of $\succsim_{i}^{* *}$ leads to $\left(y_{i}, y_{i}\right) \succ_{i}^{* *}\left(x_{i}, y_{i}\right)$, a contradiction. Hence, $P_{i}$ is asymmetric.

Two cases arise:

- If attribute $i \in N$ is degenerate then $\succ_{i}^{*}=\emptyset$. Hence $\succsim_{i}^{* *}$ has only equivalence class and $P_{i}$ is empty. We clearly have $\left[x_{i} I_{i} y_{i}\right.$ and $\left.z_{i} I_{i} w_{i}\right]$ $\Rightarrow\left(x_{i}, y_{i}\right) \sim_{i}^{* *}\left(z_{i}, w_{i}\right)$.

- If attribute $i \in N$ is influent, we claim that $P_{i}$ is non empty and that $\succsim_{i}^{* *}$ has exactly three equivalence class. Indeed, $\succsim_{i}^{*}$ being complete, there are $z_{i}, w_{i}, x_{i}, y_{i} \in X_{i}$ such that $\left(x_{i}, y_{i}\right) \succ_{i}^{*}\left(z_{i}, w_{i}\right)$. Since $\succsim_{i}^{* *}$ is complete, this implies $\left(x_{i}, y_{i}\right) \succ_{i}^{* *}\left(z_{i}, w_{i}\right)$. If $\left(x_{i}, y_{i}\right) \succ_{i}^{* *}\left(y_{i}, y_{i}\right)$ then $x_{i} P_{i} y_{i}$. If not, then $\left(y_{i}, y_{i}\right) \succsim_{i}^{* *}\left(x_{i}, y_{i}\right)$ and $\succeq_{i}^{* *}$ being a weak order, we obtain 
$\left(y_{i}, y_{i}\right) \succ_{i}^{* *}\left(z_{i}, w_{i}\right)$. Using the definition of $\succsim_{i}^{* *}$, this clearly implies $\left(w_{i}, z_{i}\right) \succ_{i}^{* *}\left(y_{i}, y_{i}\right)$. Since $\succ$ is independent, we have $\left(y_{i}, y_{i}\right) \sim_{i}^{* *}\left(z_{i}, z_{i}\right)$. Thus $\left(w_{i}, z_{i}\right) \succ_{i}^{* *}\left(z_{i}, z_{i}\right)$ so that $w_{i} P_{i} z_{i}$. Therefore $P_{i}$ is not empty.

Since $\succsim_{i}^{* *}$ has at most three distinct equivalence classes and $x_{i} P_{i} y_{i} \Leftrightarrow$ $\left(x_{i}, y_{i}\right) \succ_{i}^{* *}\left(y_{i}, y_{i}\right) \Leftrightarrow\left(y_{i}, y_{i}\right) \succ_{i}^{* *}\left(y_{i}, x_{i}\right)$, we conclude that $\succsim_{i}^{* *}$ has exactly three distinct equivalence classes. Therefore, $x_{i} P_{i} y_{i}$ implies that $\left(x_{i}, y_{i}\right)$ belongs to the first equivalence class of $\succsim_{i}^{* *}$. This implies $\left[x_{i} P_{i} y_{i}\right.$ and $\left.z_{i} P_{i} w_{i}\right] \Rightarrow\left(x_{i}, y_{i}\right) \sim_{i}^{* *}\left(z_{i}, w_{i}\right)$. Similarly, it is easy to prove that $\left[x_{i} I_{i} y_{i}\right.$ and $\left.z_{i} I_{i} w_{i}\right] \Rightarrow\left(x_{i}, y_{i}\right) \sim_{i}^{* *}\left(z_{i}, w_{i}\right)$.

Therefore, $[P(x, y)=P(z, w)$ and $P(y, x)=P(w, z)]$ implies $\left[\left(z_{i}, w_{i}\right) \sim_{i}^{* *}\right.$ $\left(x_{i}, y_{i}\right)$, for all $\left.i \in N\right]$. From part $i v$. of lemma 3 we obtain:

$$
[P(x, y)=P(z, w) \text { and } P(y, x)=P(w, z)] \Rightarrow[x \succ y \Leftrightarrow z \succ w] .
$$

Using the nondecreasingness of $F$ it is easy to prove that:

$$
[P(x, y) \subseteq P(z, w) \text { and } P(y, x) \supseteq P(w, z)] \Rightarrow[x \succ y \Rightarrow z \succ w] .
$$

Consider any two disjoint subsets $A, B \subset N$ and let:

$A \triangleright B \Leftrightarrow$

$[x \succ y$, for some $x, y \in X$ such that $P(x, y)=A$ and $P(y, x)=B]$

Equations (5) and (6) show that $\triangleright$ is asymmetric and monotonic. In view of (5), it is clear that:

$$
x \succ y \Leftrightarrow P(x, y) \triangleright P(y, x)
$$

The binary relation $\succ$ is said to be coarse on attribute $i \in N\left(C_{i}\right)$ if,

$$
\left.\begin{array}{l}
\left(x_{i}, y_{i}\right) \succ_{i}^{*}\left(y_{i}, y_{i}\right) \\
\text { or } \\
\left(y_{i}, y_{i}\right) \succ_{i}^{*}\left(y_{i}, x_{i}\right)
\end{array}\right\} \Rightarrow\left\{\begin{array}{l}
\operatorname{Not}\left[\left(z_{i}, w_{i}\right) \succ_{i}^{*}\left(x_{i}, y_{i}\right)\right] \\
\text { and } \\
\operatorname{Not}\left[\left(y_{i}, x_{i}\right) \succ_{i}^{*}\left(w_{i}, z_{i}\right)\right]
\end{array}\right.
$$

for all $x_{i}, y_{i}, z_{i}, w_{i} \in X_{i}$.

Intuitively, a relation is coarse on attribute $i \in N$ if as soon as a given preference difference is larger than a null preference difference then it cannot be beaten and its "opposite" cannot beat any preference difference. Similarly, if a preference difference is smaller than a null preference difference, then it cannot beat any preference difference and its "opposite" cannot be beaten. It is not difficult to find relations $\succ$ satisfying $C_{i}$ but not $C_{j}$ for $j \neq i$. We say that $\succ$ is coarse $(C)$ if it is coarse on all $i \in N$. 
Proposition 2 We have:

i. C, ARC1 and ARC2 are independent conditions,

ii. if $\succ$ satisfies $A R C 1$ and $A R C 2$ then $[C$ holds $] \Leftrightarrow\left[\succsim_{i}^{* *}\right.$ has at most three equivalence classes, for all $i \in N]$.

\section{Proof of proposition 2}

$i$. Using a nontrivial additive utility model, it is easy to build examples of relations satisfying $A R C 1$ and $A R C 2$ and violating $C$. The two examples used in the proof of part $i$. of lemma 1 show that there are asymmetric relations $\succ$ satisfying $C$ and $A R C 1$ (resp. $A R C 2$ ) but violating $A R C 2$ (resp. $A R C 1)$.

ii. Suppose that $A R C 1$ and $A R C 2$ hold. Let us show that $\left[\succsim_{i}^{* *}\right.$ has at most three equivalence classes, for all $i \in N] \Rightarrow C$. Suppose that $C$ is violated with $\left(x_{i}, y_{i}\right) \succ_{i}^{*}\left(y_{i}, y_{i}\right)$. We have either $\left(z_{i}, w_{i}\right) \succ_{i}^{*}\left(x_{i}, y_{i}\right)$ or $\left(y_{i}, x_{i}\right) \succ_{i}^{*}\left(w_{i}, z_{i}\right)$, for some $z_{i}, w_{i} \in X_{i}$. Since $\succ_{i}^{*} \subseteq \succ_{i}^{* *}$ and $\succsim_{i}^{* *}$ is a reversible weak order, it is easy to see that either case implies that $\succsim_{i}^{* *}$ has at least five equivalence classes. The case $\left(y_{i}, y_{i}\right) \succ_{i}^{*}\left(y_{i}, x_{i}\right)$ is similar.

Let us now show that $C \Rightarrow\left[\succsim_{i}^{* *}\right.$ has at most three equivalence classes, for all $i \in N]$. Suppose that $\left(x_{i}, y_{i}\right) \succ_{i}^{* *}\left(y_{i}, y_{i}\right)$ so that either $\left(x_{i}, y_{i}\right) \succ_{i}^{*}$ $\left(y_{i}, y_{i}\right)$ or $\left(y_{i}, y_{i}\right) \succ_{i}^{*}\left(y_{i}, x_{i}\right)$. In either case, $C$ implies, for all $z_{i}, w_{i} \in X_{i}$, $\left(x_{i}, y_{i}\right) \succsim_{i}^{*}\left(z_{i}, w_{i}\right)$ and $\left(w_{i}, z_{i}\right) \succsim_{i}^{*}\left(y_{i}, x_{i}\right)$ so that $\left(x_{i}, y_{i}\right) \succsim_{i}^{* *}\left(z_{i}, w_{i}\right)$. Therefore if $\left(x_{i}, y_{i}\right) \succ_{i}^{* *}\left(y_{i}, y_{i}\right)$ then $\left(x_{i}, y_{i}\right) \succsim_{i}^{* *}\left(z_{i}, w_{i}\right)$ for all $z_{i}, w_{i} \in X_{i}$. Similarly, it is easy to prove that $\left(y_{i}, y_{i}\right) \succ_{i}^{* *}\left(x_{i}, y_{i}\right)$ implies $\left(z_{i}, w_{i}\right) \succsim_{i}^{* *}\left(x_{i}, y_{i}\right)$ for all $z_{i}, w_{i} \in X_{i}$. This implies that $\succsim_{i}^{* *}$ has at most three equivalence classes.

Combining theorem 2 with proposition 2 therefore leads to a characterization of strict concordance relations. We have:

Theorem 3 Let $\succ$ be a binary relation on a finite set $X=\prod_{i=1}^{n} X_{i}$. The following are equivalent:

i. $\succ$ is asymmetric and satisfies $A R C 1, A R C 2$ and $C$,

ii. $\succ$ is a strict concordance relation.

It is interesting to observe that this characterization uses two conditions ( $A R C 1$ and $A R C 2$ ) that are far from being specific to concordance methods. 
In fact, as shown in [BP00], these conditions can be considered as the building blocks of most conjoint measurement models. The specificity of strict concordance relations lies in condition $C$ which imposes that only a very rough differentiation of preference differences is possible on each attribute. Clearly, $C$ should not be viewed as a condition with normative content. In line with $\left[\mathrm{BPP}^{+} 93\right]$, it is simply used here as a means to point out the specificities of strict concordance relations. It is easy, but not very informative, to reformulate $C$ in terms of $\succ$. We leave to the reader the easy proof of the following:

Proposition 3 If $\succ$ satisfies $A R C 1$ and $A R C 2$ then $C$ holds if and only if, for all $i \in N$, all $x_{i}, y_{i} \in X_{i}$, all $x_{-i}, y_{-i} \in X_{-i}$ and all $z, w \in X$,

$$
\begin{aligned}
& \left.\begin{array}{l}
\left(x_{i}, x_{-i}\right) \succ\left(y_{i}, y_{-i}\right) \text { and } \operatorname{Not}\left[\left(y_{i}, x_{-i}\right) \succ\left(y_{i}, y_{-i}\right)\right] \\
\operatorname{Not}\left[\left(y_{i}, x_{-i}\right) \succ\left(x_{i}, y_{-i}\right)\right] \text { and }\left(y_{i}, x_{-i}\right) \succ\left(y_{i}, y_{-i}\right)
\end{array}\right\} \Rightarrow \\
& \left\{\begin{array}{l}
z \succ w \Rightarrow\left(x_{i}, z_{-i}\right) \succ\left(y_{i}, w_{-i}\right) \\
\text { and } \\
\left(y_{i}, w_{-i}\right) \succ\left(x_{i}, z_{-i}\right) \Rightarrow w \succ z
\end{array}\right.
\end{aligned}
$$

\section{Discussion and remarks}

\subsection{Strict concordance relations and noncompensatory preferences}

It has long been thought [Bou86, BV86] that the notion of noncompensatory preferences, as defined in [Fis76], provided the adequate framework for the characterization of strict concordance relations. We think that the framework provided by model (M) is more general and adequate for doing so.

P.C. FishBURN's definition of noncompensatory preferences [Fis76] starts with an asymmetric binary relation $\succ$ on $X=\prod_{i=1}^{n} X_{i}$. Let $\succ(x, y)=\{i$ : $\left.x_{i} \succ_{i} y_{i}\right\}$ and $\sim(x, y)=\left\{i: x_{i} \sim_{i} y_{i}\right\}$. It is clear that, for all $x, y \in X$, $\succ(x, y) \cap \succ(y, x)=\emptyset, \sim(x, y)=\sim(y, x)$ and $\succ(x, y) \cap \sim(x, y)=\emptyset$. Note that, in general, it is not true that $\succ(x, y) \cup \sim(x, y) \cup \succ(y, x)=N$ since the relations $\succsim_{i}$ might be incomplete. 


\section{Definition 2 ([Fis76])}

The binary relation $\succ$ is said to be noncompensatory (in the asymmetric sense) if:

$$
\left.\begin{array}{l}
\succ(x, y)=\succ(z, w) \\
\succ(y, x)=\succ(w, z)
\end{array}\right\} \Rightarrow[x \succ y \Leftrightarrow z \succ w],
$$

for all $x, y, z, w \in X$.

Hence, when $\succ$ is noncompensatory, the preference between $x$ and $y$ only depends on the subsets of attributes favoring $x$ or $y$. It does not depend on preference differences between the various levels on each attribute besides the distinction between "positive", "negative" and "neutral" attributes. Some useful properties of noncompensatory preferences are summarized in the following:

Proposition 4 If an asymmetric relation $\succ$ is noncompensatory, then:

i. $\succ$ is independent,

ii. $x_{i} \sim_{i} y_{i}$ for all $i \in N \Rightarrow x \sim y$,

iii. $x_{j} \succ_{j} y_{j}$ for some $j \in N$ and $x_{i} \sim_{i} y_{i}$ for all $i \in N \backslash\{j\} \Rightarrow x \succ y$,

$i v$. all influent attributes are essential.

\section{Proof of proposition 4}

$i$. Since $\sim_{i}$ is reflexive by construction, the definition of noncompensation implies that $\succ$ is independent for $N \backslash\{i\}$. Hence, $\succ$ is independent.

ii. Suppose that $x_{i} \sim_{i} y_{i}$ for all $i \in N$ and $x \succ y$. Since $\succ$ is noncompensatory and $\sim_{i}$ is reflexive, this would lead to $x \succ x$, contradicting the asymmetry of $\succ$.

iii. By definition, $x \succ_{i} y \Leftrightarrow\left[\left(x_{i}, z_{-i}\right) \succ\left(y_{i}, z_{-i}\right)\right.$ for all $\left.z_{-i} \in X_{-i}\right]$. Since $\sim_{i}$ is reflexive, the desired conclusion follows from the definition of noncompensation.

$i v$. Attribute $i \in N$ being influent, there are $x_{i}, y_{i}, z_{i}, w_{i} \in X_{i}$ and $x_{-i}, y_{-i}$ $\in X_{-i}$ such that $\left(x_{i}, x_{-i}\right) \succ\left(y_{i}, y_{-i}\right)$ and $\operatorname{Not}\left[\left(z_{i}, x_{-i}\right) \succ\left(w_{i}, y_{-i}\right)\right]$. In view of $\mathrm{NC}$, it is impossible that $x_{i} \sim_{i} y_{i}$ and $z_{i} \sim_{i} w_{i}$. Hence attribute $i$ is essential. 
It is not difficult to see that there are strict concordance relations violating all conditions in proposition 4 except independence. Examples of such situations are easily built using a strict concordance relation defined by:

$$
x \mathcal{P} y \Leftrightarrow \sum_{i \in P(x, y)} w_{i}>\sum_{j \in P(y, x)} w_{j}+\varepsilon
$$

where $\varepsilon>0, w_{i}>0$ for all $i \in N$. Letting $w_{j}<\varepsilon$ on some attributes easily leads to the desired conclusions (e.g. an attribute such that $w_{j}<\varepsilon$ is not essential but may well be influent).

Hence basing the analysis of concordance relations on condition NC leads to a somewhat narrow view of concordance relations. Noncompensation implies that all influent attributes are essential, whereas this is not the case for strict concordance relations.

When $\succ$ is noncompensatory, it is entirely defined by the partial preference relations on each attribute and an asymmetric importance relation between disjoint subsets of attributes. We formalize this idea below using a strengthening of NC including an idea of monotonicity (see also [FPng]).

\section{Definition 3}

The binary relation $\succ$ is said to be monotonically noncompensatory (in the asymmetric sense) if:

$$
\left.\begin{array}{l}
\succ(x, y) \subseteq \succ(z, w) \\
\succ(y, x) \supseteq \succ(w, z)
\end{array}\right\} \Rightarrow[x \succ y \Rightarrow z \succ w]
$$

for all $x, y, z, w \in X$.

It is clear that $\mathrm{MNC} \Rightarrow \mathrm{NC}$. We have:

Proposition 5 The following are equivalent:

$i . \succ$ is a strict concordance relation in which all attributes are essential,

ii. $\succ$ is an asymmetric binary relation satisfying $M N C$.

\section{Proof of proposition 5}

$i$. $\Rightarrow i i$. Since each attribute is essential, it is easy to see that $\{i\} \triangleright \emptyset$ so that $P_{i}=\succ_{i}$. The conclusion therefore follows. 
ii. $\Rightarrow i$. Letting $P_{i}=\succ_{i}$ and defining $\triangleright$ by:

$[A \triangleright B] \Leftrightarrow$

$[x \succ y$, for some $x, y \in X$ such that $\succ(x, y)=A$ and $\succ(y, x)=B]$.

easily leads to the desired conclusion.

Therefore, all asymmetric relations satisfying MNC are strict concordance relations and the converse is true as soon as all attributes are supposed to be essential. In our nontransitive setting, assuming that all attributes are essential is far from being an innocuous hypothesis. It implies that the relations $P_{i}$ used to show that $\succ$ is a strict concordance relation must coincide with the relations $\succ_{i}$ deduced from $\succ$ by independence. Equation (7) shows that this is indeed restrictive.

Therefore, it seems that the use of $\mathrm{NC}$ or MNC for the analysis of strict concordance relations:

$i$. leads to a somewhat narrow view of strict concordance relations excluding all relations in which attributes may be influent without being essential,

ii. does not allow to point out the specific features of strict concordance relations within a general framework of conjoint measurement (conditions NC and MNC are indeed quite different from the classical cancellation conditions used in most conjoint measurement models, and most importantly, the additive utility model [KLST71, Deb60, Fis70, Wak89]),

iii. amounts to using very strong conditions (see the simple proof of proposition 5).

\subsection{Transitivity of partial preferences}

Our definition of strict concordance relations (3) does not require the relations $P_{i}$ to possess any remarkable property besides asymmetry. This is at variance with what is done in most outranking methods which use relations $P_{i}$ being strict semiorders. It might be thought that this additional condition might lead to an improved characterization of strict concordance relations. However, it is shown in [BP01] that the various conditions that can be used 
to decompose the functions $p_{i}$ in model $(\mathrm{M})$ so as to consider preference differences which are governed by an underlying weak order (as in the case of semiorders) are independent from $A R C 1$ and $A R C 2$. These additional conditions are furthermore independent from $C$. Therefore there is little hope to arrive at a more powerful characterization adding the hypothesis that $P_{i}$ are strict semiorders.

\subsection{Transitivity of concordance relations and Arrow's theorem}

One advantage of the use of conditions $\mathrm{NC}$ and $\mathrm{MNC}$ is that they allow to clearly understand the conditions under which $\succ$ may possess "nice transitivity properties". This is not surprising since NC (resp. MNC) is very much like a "single profile" analogue of Arrow's Independence of Irrelevant Alternatives [Arr63] (resp. the NIM condition used in [Sen86]). Therefore, as soon as the structure of $X$ is sufficiently rich, imposing nice transitivity properties on a noncompensatory relation $\succ$ leads to a very uneven distribution of "power" between the various attributes [Fis76, Bou92].

It is not difficult to see that similar results hold with strict concordance relations. We briefly present below one such result as an example, extending to our case a single profile result due to [Wey83]. Other results in [Fis76, Bou92, PF99] can be reformulated in a similar way.

Proposition 6 Let $\succ$ be a nonempty strict concordance relation on a finite set $X=\prod_{i=1}^{n} X_{i}$. Suppose that $\succ$ has been obtained using, on each $i \in N$, a relation $P_{i}$ for which there are $a_{i}, b_{i}, c_{i} \in X_{i}$ such that $a_{i} P_{i} b_{i}, b_{i} P_{i} c_{i}$ and $a_{i} P_{i} c_{i}$. Then, if $\succ$ is transitive, it has an oligarchy, i.e. there is a unique nonempty $O \subseteq N$ such that, for all $x, y \in X$ :

- $x_{i} P_{i} y_{i}$ for all $i \in O \Rightarrow x \succ y$,

- $x_{i} P_{i} y_{i}$ for some $i \in O \Rightarrow \operatorname{Not}[y \succ x]$.

\section{Proof of proposition 6}

We say that a nonempty set $J \subseteq N$ is:

- decisive if, for all $x, y \in X,\left[x_{i} P_{i} y_{i}\right.$ for all $\left.i \in J\right] \Rightarrow x \succ y$,

- semi-decisive if, for all $x, y \in X,\left[x_{i} P_{i} y_{i}\right.$ for all $\left.i \in J\right] \Rightarrow N o t[y \succ x]$, 
Hence, an oligarchy $O$ is a decisive set such that all $\{i\} \subseteq O$ are semi-decisive.

Since $\succ$ is a strict concordance relation, it is easy to prove that:

$$
\begin{aligned}
{[P(x, y)=J, P(y, x)=} & N \backslash J \text { and } x \succ y, \text { for some } x, y \in X] \\
& \Rightarrow J \text { is decisive, }
\end{aligned}
$$

and

$$
\begin{aligned}
{[P(x, y)=J, P(y, x)} & =N \backslash J \text { and } N o t[y \succ x], \text { for some } x, y \in X] \\
& \Rightarrow J \text { is semi-decisive. }
\end{aligned}
$$

Since $\succ$ is nonempty, we have, for all $x, y \in X$ :

$$
x_{i} P_{i} y_{i} \text { for all } i \in N \Rightarrow x \succ y
$$

so that $N$ is decisive.

Since $N$ is finite, there exists (at least) one decisive set of minimal cardinality. Let $J$ be one of them. We have $\left[x_{i} P_{i} y_{i}\right.$ for all $\left.i \in J\right] \Rightarrow x \succ y$. If $|J|=1$, then the conclusion follows. If not, consider $i \in J$ and use the elements $a_{i}, b_{i}, c_{i} \in X_{i}$ such that $a_{i} P_{i} b_{i}, b_{i} P_{i} c_{i}$ and $a_{i} P_{i} c_{i}$ to build the following alternatives in $X$ :

\begin{tabular}{cccc}
\hline & $\{i\}$ & $J \backslash\{i\}$ & $N \backslash J$ \\
\hline$a$ & $c_{i}$ & $a_{j}$ & $b_{\ell}$ \\
$b$ & $a_{i}$ & $b_{j}$ & $c_{\ell}$ \\
$c$ & $b_{i}$ & $c_{j}$ & $a_{\ell}$ \\
\hline
\end{tabular}

$J$ being decisive, we have $b \succ c$. If $a \succ c$, then $J \backslash\{i\}$ is decisive, violating the fact that $J$ is a decisive set of minimal cardinality. We thus have $\operatorname{Not}[a \succ c]$ and the transitivity of $\succ$ leads to $\operatorname{Not}[a \succ b]$. This shows that $\{i\}$ is semi-decisive. Therefore all singletons in $J$ are semi-decisive.

The proof is completed observing that $J$ is necessarily unique. In fact suppose that there are two sets $J$ and $J^{\prime}$ with $J \neq J^{\prime}$ satisfying the desired conclusion. We use the elements $a_{i}, b_{i} \in X_{i}$ such that $a_{i} P_{i} b_{i}$ to build the following alternatives in $X$ :

\begin{tabular}{cccc}
\hline & $J$ & $J^{\prime} \backslash J$ & $N \backslash\left[J \cup J^{\prime}\right]$ \\
\hline$d$ & $a_{j}$ & $b_{k}$ & $a_{\ell}$ \\
$e$ & $b_{j}$ & $a_{k}$ & $a_{\ell}$ \\
\hline
\end{tabular}

We have, by construction, $e \succ d$ and $\operatorname{Not}[e \succ d]$, a contradiction. 


\subsection{A possible definition of the degree of compensation of a binary relation}

Within the general framework of model (M), our results show that relations $\succsim_{i}^{* *}$ seem central to understand the possibility of trade-offs between attributes.

We therefore tentatively suggest that the "degree of compensation" of an asymmetric binary relation $\succ$ on a finite set $X=X_{1} \times X_{2} \times \cdots \times X_{n}$ satisfying $A R C 1$ and $A R C 2$ should be linked to the number $c_{i}^{* *}$ of distinct equivalence classes of $\succsim_{i}^{* *}$ on each attribute. We have $c_{i}^{* *} \leq 3$, for all $i \in N$ if and only if $\succ$ is a strict concordance relation (see theorem 2). Letting $\left|X_{i}\right|=n_{i}, c_{i}^{* *}$ can be as large as $n_{i} \times\left(n_{i}-1\right)+1$ when $\succ$ is representable in an additive utility model or an additive difference model.

A reasonable way of obtaining an overall measure of the degree of compensation of $\succ$ consists in taking:

$$
c^{* *}=\max _{i=1,2, \ldots, n} c_{i}^{* *} .
$$

This leads to $c^{* *} \leq 3$ iff $\succ$ is a strict concordance relation.

An aggregation technique can produce a whole set of binary relations on a finite set $X=X_{1} \times X_{2} \times \cdots \times X_{n}$ depending on the choice of various parameters. We suggest to measure the degree of compensation of an aggregation technique (always producing asymmetric binary relations satisfying $A R C 1$ and $A R C 2$ ) as the maximum value of $c^{* *}$ taken over the set of binary relations on $X$ that can be obtained with this technique.

Since an additive utility model can be used to represent lexicographic preferences on finite sets, the choice of the operator "max" should be no surprise: using "min" would have led to a similar measure for methods based on concordance and methods using additive utilities and it is difficult to conceive an "averaging" operator that would be satisfactory. Using such a definition, aggregation methods based on concordance have the minimal possible measure (i.e., 3), whereas the additive utility model has a much higher value (the precise value depends on $n_{i}$ and $n$ ). It should finally be noted that our proposals are at variance with [Roy96a] who uses a more topological approach to the idea of compensation.

The validation of our proposals and their extension to sets of arbitrary cardinality clearly call for future research. 


\subsection{Discordance}

An immediate generalization of definition 1 is the following:

\section{Definition 4 (Strict concordance-discordance relations)}

A binary relation $\mathcal{P}$ on $X$ is said to be a strict concordance-discordance relation if there are:

- an asymmetric binary relation $\triangleright$ between disjoint subsets of $N$ that is monotonic and,

- asymmetric binary relations $P_{i}$ and $V_{i}$ such that $V_{i} \subseteq P_{i}$ on each $X_{i}$ $(i=1,2, \ldots, n)$,

such that, for all $x, y \in Y$ :

$$
x \mathcal{P} y \Leftrightarrow\left[P(x, y) \triangleright P(y, x) \text { and }\left(\operatorname{Not}\left[y_{j} V_{j} x_{j}\right] \text {, for all } j \in P(y, x)\right)\right],
$$

where $P(x, y)=\left\{i \in N: x_{i} P_{i} y_{i}\right\}$.

The only attempt at a characterization of discordance effects in outranking methods we are aware of is [BV86]. It is based on an extension of NC allowing to have $x \succ y$ and $\operatorname{Not}[z \succ w]$ when $\succ(x, y)=\succ(z, w)$ and $\succ(y, x)=\succ(w, z)$. This analysis, based on NC, is therefore subject to the criticisms made in section 5.1 (let us also mention that such an analysis cannot be easily extended to outranking methods producing binary relations that are not necessarily asymmetric, e.g. ELECTRE I; in that case, discordance effects may well create situations in which $x \succ y$ and $w \succ z$ while $P(x, y)=P(z, w)$ and $P(y, x)=P(w, z)$, through destroying what would have otherwise been indifference situations $x \sim y$ and $z \sim w)$. Furthermore, the above-mentioned extension of $\mathrm{NC}$ is far from capturing the essence of discordance effects, i.e. the fact that they occur attribute by attribute, leaving no room for possible interactions between negative preference differences. The prevention of such interactions has led to the introduction of rather ad hoc axioms in [BV86].

It is not difficult to see that strict concordance-discordance relations always satisfy $A R C 1$ and $A R C 2$ with relations $\succsim_{i}^{* *}$ having at most 5 distinct equivalence classes (compared to strict concordance relations, the two new classes correspond to "very large" positive and negative preference differences). However, model (M) is clearly not well adapted to prevent the possibility of interactions between very large negative preference differences, as 
is the case for discordance effects. Simple examples show that if the class of relations $\succ$ satisfying $A R C 1$ and $A R C 2$ with relations $\succsim_{i}^{* *}$ having at most 5 equivalence classes contains all strict concordance-discordance relations, it contains many more relations. This clearly calls for future research. We nevertheless summarize our observations in the following:

\section{Proposition 7}

i. If $\succ$ is a strict concordance-discordance relation then $\succ$ satisfies model (M) with all relations $\succsim_{i}^{* *}$ having at most 5 distinct equivalence classes.

ii. There are relations $\succsim$ satisfying model $(M)$ with all relations $\succsim_{i}^{* *}$ having at most 5 equivalence classes which are not strict concordancediscordance relations.

\section{Proof of proposition 7}

$i$. Given the properties of model (M), the claim will be proven if we build a representation of $\succ$ in model (M) with functions $p_{i}$ taking only five distinct values. Define $p_{i}$ as:

$$
p_{i}\left(x_{i}, y_{i}\right)=\left\{\begin{array}{l}
2 \text { if } x_{i} V_{i} y_{i} \\
1 \text { if } x_{i} P_{i} y_{i} \text { and } \operatorname{Not}\left[x_{i} V_{i} y_{i}\right] \\
0 \text { if } x_{i} I_{i} y_{i} \\
-1 \text { if } y_{i} P_{i} x_{i} \text { and } \operatorname{Not}\left[y_{i} V_{i} x_{i}\right] \\
-2 \text { if } y_{i} V_{i} x_{i}
\end{array}\right.
$$

Since $V_{i}$ and $P_{i}$ are asymmetric and $V_{i} \subseteq P_{i}$, the function $p_{i}$ is well-defined and skew-symmetric.

Define $F$ letting:

$$
F\left(p_{1}\left(x_{1}, y_{1}\right), p_{2}\left(x_{2}, y_{2}\right), \ldots, p_{n}\left(x_{n}, y_{n}\right)\right)=\left\{\begin{array}{l}
1 \text { if } x \succ y, \\
-1 \text { if } y \succ x, \\
0 \text { otherwise. }
\end{array}\right.
$$

Using the definition of a strict concordance-discordance relation, it is routine to show that $F$ is well-defined, odd and nondecreasing.

ii. Using an additive utility model, it is easy to build examples of relations having a representation in model (M) with all relations $\succsim_{i}^{* *}$ having at most 5 equivalence classes which are not strict concordance-discordance relations. 


\subsection{Discussion}

The main contribution of this paper was to propose a characterization of strict concordance relations within the framework of a general model for nontransitive conjoint measurement. This characterization allows to show the common features between various conjoint measurement models and to isolate the specific feature of strict concordance relations, i.e. the option not to distinguish a rich preference difference relation on each attribute. It was shown to be more general than previous ones based on NC or MNC.

Although we restricted our attention to asymmetric relations, it is not difficult to extend our analysis, using the results in [BP00], to cover the reflexive case studied in [FPng] in which:

$$
x \mathcal{S} y \Leftrightarrow[S(x, y) \unrhd S(y, x)]
$$

where $\mathcal{S}$ is a reflexive binary relation on $X, S_{i}$ is a complete binary relation on $X_{i}, \unrhd$ is a reflexive binary relation on $2^{N}$ and $S(x, y)=\left\{i \in N: x_{i} S_{i} y_{i}\right\}$.

Further research on the topics discussed in this paper could involve:

- the extension of our results to cover the case of an homogeneous Cartesian product, which includes the important case of decision under uncertainty. "Ordinal" models for decision under uncertainty (e.g. lifting rules) have been characterized in [PF99] using variants of $\mathrm{NC}$ and MNC. It appears that our analysis can be easily extended to cover that case, see [BPP00].

- a deeper study of discordance effects within model (M). Such a work could possibly allow for a characterization of strict concordance-discordance relations in our conjoint measurement framework.

- a study of various variants of model $(\mathrm{M})$ following the approach in [BP00, BP01].

\section{References}

[Arr63] K.J. Arrow. Social choice and individual values. Wiley, New York, 2nd edition, 1963. 
[Bou86] D. Bouyssou. Some remarks on the notion of compensation in MCDM. European Journal of Operational Research, 26:150-160, 1986.

[Bou92] D. Bouyssou. On some properties of outranking relations based on a concordance-discordance principle. In L. Duckstein, A. Goicoechea, and S. Zionts, editors, Multiple criteria decision making, pages 93-106. Springer-Verlag, Berlin, 1992.

[Bou96] D. Bouyssou. Outranking relations: Do they have special properties? Journal of Multi-Criteria Decision Analysis, 5:99-111, 1996.

[Bou01] D. Bouyssou. Outranking methods. In C.A. Floudas and P.M. Pardalos, editors, Encyclopedia of optimization. Kluwer, 2001.

[BP00] D. Bouyssou and M. Pirlot. Non transitive decomposable conjoint measurement: General representation of non transitive preferences on product sets. Working Paper, 2000.

[BP01] D. Bouyssou and M. Pirlot. 'Additive difference' models without additivity and subtractivity. Working Paper, 2001.

$\left[\mathrm{BPP}^{+} 93\right]$ D. Bouyssou, P. Perny, M. Pirlot, A. Tsoukiàs, and Ph Vincke. A manifesto for the new MCDM era. Journal of Multi-Criteria Decision Analysis, 2:125-127, 1993.

[BPP00] D. Bouyssou, P. Perny, and M. Pirlot. Nontransitive decomposable conjoint measurement as a general framework for MCDM and decision under uncertainty. Communication to EURO XVII, Budapest, Hungary, 16-19 July, 2000.

[BV86] D. Bouyssou and J.-C. Vansnick. Noncompensatory and generalized noncompensatory preference structures. Theory and Decision, 21:251-266, 1986.

[Deb60] G. Debreu. Topological methods in cardinal utility theory. In K.J. Arrow, S. Karlin, and P. Suppes, editors, Mathematical methods in the social sciences, pages 16-26. Stanford University Press, 1960. 
[Fis70] P.C. Fishburn. Utility theory for decision-making. Wiley, New York, 1970.

[Fis76] P.C. Fishburn. Noncompensatory preferences. Synthese, 33:393403, 1976.

[Fis90a] P.C. Fishburn. Additive non-transitive preferences. Economic Letters, 34:317-321, 1990.

[Fis90b] P.C. Fishburn. Continuous nontransitive additive conjoint measurement. Mathematical Social Sciences, 20:165-193, 1990.

[Fis91] P.C. Fishburn. Nontransitive additive conjoint measurement. Journal of Mathematical Psychology, 35:1-40, 1991.

[Fis92] P.C. Fishburn. Additive differences and simple preference comparisons. Journal of Mathematical Psychology, 36:21-31, 1992.

[FPng] H. Fargier and P. Perny. Modélisation des préférences par une règle de concordance généralisée. In B. Roy et al., editor, $A M C D A, S e$ lected papers from the 49th and 50th meetings of the EURO Working Group on Multicriteria Aid for Decisions. European Union, forthcoming.

[KLST71] D.H. Krantz, R.D. Luce, P. Suppes, and A. Tversky. Foundations of measurement, volume 1: Additive and polynomial representations. Academic Press, New York, 1971.

[KR76] R.L. Keeney and H. Raiffa. Decisions with multiple objectives: Preferences and value tradeoffs. Wiley, 1976.

[PF99] P. Perny and H. Fargier. Qualitative decision models under uncertainty without the commensurability assumption. In K.B. Laskey and H. Prade, editors, Proceedings of Uncertainty in Artificial Intelligence, pages 188-195. Morgan Kaufmann Publishers, 1999.

[RB93] B. Roy and D. Bouyssou. Aide multicritère à la décision : Méthodes et cas. Economica, Paris, 1993.

[Roy68] B. Roy. Classement et choix en présence de points de vue multiples (la méthode ELECTRE). RIRO, 2:57-75, 1968. 
[Roy91] B. Roy. The outranking approach and the foundations of ELECTRE methods. Theory and Decision, 31:49-73, 1991.

[Roy96a] B. Roy. Les logiques compensatoires et les autres. Research paper \# 16, LAMSADE, Université de Paris-Dauphine, 1996.

[Roy96b] B. Roy. Multicriteria methodology for decision aiding. Kluwer, Dordrecht, 1996. Original version in French: "Méthodologie multicritère d'aide à la décision", Economica, Paris, 1985.

[Sen86] A.K. Sen. Social choice theory. In K.J. Arrow and M.D. Intriligator, editors, Handbook of mathematical economics, volume 3, pages 1073-1181. North-Holland, Amsterdam, 1986.

[Tve69] A. Tversky. Intransitivity of preferences. Psychological Review, $76: 31-48,1969$.

[Van86] J.-C. Vansnick. On the problems of weights in MCDM (the noncompensatory approach). European Journal of Operational Research, 24:288-294, 1986.

[Van90] D. Vanderpooten. The construction of prescriptions in outranking methods. In C.A. Bana e Costa, editor, Readings in multiple criteria decision aid, pages 184-215. Springer Verlag, Berlin, 1990.

[Vin91] K. Vind. Independent preferences. Journal of Mathematical Economics, 20:119-135, 1991.

[Vin92] Ph. Vincke. Multi-criteria decision aid. Wiley, New York, 1992. Original version in French: "L'aide multicritère à la décision", Éditions de l'Université de Bruxelles-Éditions Ellipses, Brussels, 1989.

[Vin99] Ph. Vincke. Outranking approach. In T. Gal, T. Stewart, and T. Hanne, editors, Multicriteria decision making, Advances in MCDM models, algorithms, theory and applications, pages 11.111.29. Kluwer, 1999.

[Wak88] P.P. Wakker. Derived strength of preference relations on coordinates. Economic Letters, 28:301-306, 1988. 
[Wak89] P.P. Wakker. Additive representations of preferences - A new foundation of decision analysis. Kluwer, Dordrecht, 1989.

[Wey83] J.A. Weymark. Arrow's theorem with quasi-orderings. Public Choice, 42:235-246, 1983. 